NASA Contractor Report 182241

\title{
Solution and Sensitivity Analysis of a Complex Transcendental Eigenproblem With Pairs of Real Eigenvalues
}

Durbha V. Murthy

The University of Toledo

Toledo, Ohio

January 1989

Prepared for

Lewis Research Center

Under Grant NAG3-742

\section{NMSA}

National Aeronautics and

Space Administration 


\title{
Solution and Sensitivity Analysis of A Complex
}

\section{Transcendental Eigenproblem with Pairs of Real}

\author{
Eigenvalues
}

\author{
Durbha V. Murthy* \\ The University of Toledo \\ Toledo, Ohio
}

SLMMARY

\begin{abstract}
This paper considers complex transcendental eigenvalue problems where one is interested in pairs of eigenvalues that are restricted to take rcal values only. Such eigenvalue problems arise in dynamic stability analysis of non-conservative physical systems - flutter analysis of aeroelastic systems, to name one example. Some available solution methods are discussed and a new method is presented. Two computational approaches are described for analytical evaluation of the sensitivities of these eigenvalues when they are dependent on other parameters. The algorithms presented are illustrated through examples.
\end{abstract}

*NASA Resident Research Associate. 


\section{Introduction}

Dynamic analysis of physical systems often requires the solution of an eigenvalue problem. Optimization of dynamic systems also requires efficient computation of the derivatives of the eigenvalues and eigenvectors. The computation of these derivatives is referred to as sensitivity analysis. Development of numerical algorithms for the evaluation of eigenvalues and eigenvectors (Wilkinson, 1965 ) and, recently, their derivatives with respect to system parameters (e.g., Murthy and Haftka, 1988), for the linear algebraic eigenvalue problem

$$
(\mathbf{A}-\lambda \mathbf{I}) \mathbf{u}=\mathbf{0}
$$

where the matrix $\mathbf{A}$ is complex non-hermitian has attracted extensive research efforts. The eigenvalues $\lambda$ and eigenvectors $\boldsymbol{u}$ for such problems are also generally complex. Some of these methods and algorithms can be directly extended to the $\lambda$-matrix (Frazer, Duncan and Collar, 1960) eigenproblem

$$
\mathbf{A}(\%) \mathbf{u}=\mathbf{0}
$$

where the elements of the complex non-hermitian matrix $\mathbf{A}$ are polynomials in the eigenvalue, $\lambda$. A discussion of the $\lambda$-matrix eigenproblem can be found in Rokne (1985). Extensive analytical results on the sensitivities of the eigenvalues and eigenvectors of eq. (2) are presented by Taylor and Kane(1975) for the special case where the polynomials are quadratic. These sensitivities were used by Fritzen and Nordmann (1982) for predicting the stability behavior of non-conservative rotor dynamic models.

In this paper, we are concerned with the solution and sensitivity analysis of the eigenvalue problem

$$
\mathbf{A}\left(\lambda_{1}, \lambda_{2}\right) \mathbf{u}=\mathbf{0}
$$

where the elements of the complex non-hermitian matrix $\mathbf{A}$ are transcendental functions of the pair, $\lambda_{1}$ and $\lambda_{2}$, which are restricted to take real values only. Comparing eq. (3) to the $\lambda$-matrix eigenproblem of eq. (2), we note two differences: 1) the pair of real numbers $\lambda_{1}$ and $\lambda_{2}$ replace the 
complex number $\lambda$ and hence play the role of eigenvalues and 2) the elements of $A$ in eq. (3) are transcendental functions of the eigenvalues instead of polynomial functions. We also note that, if $\lambda_{1}$ and $\lambda_{2}$ are allowed to take on arbitrary complex values, eq. (3) does not represent a well-defined eigenvalue problem. The cigenvectors $u$ in eq. (3) remain complex-valued.

This problem was motivated by the need to automate the flutter analysis in acroclastic optimization problems. Murthy and Kaza (1988) automated the flutter analysis procedure using the direct solution approach which results in the eigenvalue problem of the form given by eq. (3). The flutter analysis procedures of Cardani and Mantegazza(1978) and Meyer(1988) also result in eigenvalue problems of this form. Here, the matrix $\mathbf{A}$ involves the generalized stiffness, mass and aerodynamic matrices of the aeroelastic structure, which is undergoing steady-state oscillations in a state of neutral stability. The generalized stiffness, mass and aerodynamic matrices are usually computed by specialized structural and aerodynamic analysis programs which are computationally intensive. Depending upon the formulation, the eigenvalue $\lambda_{1}$ is a speed parameter such as the Mach number or the rotational speed at flutter and the eigenvalue $\lambda_{2}$ is a frequency parameter such as the reduced frequency at flutter. In aeroclastic literature, the pair $\lambda_{1}$ and $\lambda_{2}$ is called the matched flutter point and the eigenvector $\mathbf{u}$ the flutter mode. The viewing of the flutter analysis problem as a complex eigenvalue problem involving a pair of real eigenvalues was first proposed by Frazer (1946) and is called the direct solution approach. An altemate approach to flutter analysis, known as the U-g method (Bisplinghoff, Ashley and Halfman, 1955), which decomposes the solution of eq. (3) into a series of linear algebraic eigenvalue problems, has been more popular in aeroelastic literature, perhaps because of the increased physical insight it offers. However, the direct solution approach is more attractive in terms of automating the flutter analysis procedure and also more efficient, as discussed by Murthy and Kaza(1988), because it avoids troublesome eigenvalue tracking and replaces the inner-outer iteration loop required by the U-g method by a single iteration loop.

The objective of the present paper is to present (i) a solution method for calculating the eigenvalues and the eigenvectors of eq. (3) and (ii) methods for sensitivity analysis of eq. (3). The solution method was developed by Murthy and Kaza (1988) but is presented here in a more general context and with additional examples. The sensitivity analysis methods appear to be new. It is 
assumed that the computation of the matrix $A$ is much more expensive in terms of CPU time than its inversion. This is typically the case in aeroelastic applications (Murthy and Kaza, 1988).

\section{Solution Methods}

The eigenvalue problem of eq. (3) differs from those of eqs. (1) and (2) in many respects. While the conventional eigenproblems of eqs. (1) and (2) define eigenpairs, the eigenvalue problem of eq. (3) defines eigentriples, each consisting of $\lambda_{1}, \lambda_{2}$ and $\mathbf{u}$. Also, it is well-known that the number of the eigenpairs of eqs. (1) depends only on the order of the matrix and, in the case of eq. (2), on the matrix order and the highest degree of the polynomials in $\lambda$. In the case of eq. (3), the number of the eigentriples is not known in advance and could be infinite, finite or none. In addition, while the eigenvalue problems of eqs. (1) and (2) admit bi-orthogonal relationships between the left and the right eigenvectors and can be reduced to standard canonical forms, no such relationships and canonical forms are known to exist for the eigenvalue problem defined by eq. (3). This has important consequences in terms of sensitivity analysis and is discussed further in the next section.

In this paper, we will restrict our attention to those problems that satisfy the following assumptions:

- There exist real values of $\lambda_{1}$ and $\lambda_{2}$ such that eq. (3) is satisfied for some vector $\mathbf{u}$.

- For such values of $\lambda_{1}$ and $\lambda_{2}$, there exists a neighborhood in the $\lambda_{1}, \lambda_{2}$-plane in which the members of the matrix $A$ are continuously differentiable functions of $\lambda_{1}$ and $\lambda_{2}$.

These assumptions are usually satisfied in aeroelastic applications. Solution of eq. (3) consists of finding the pairs of values of $\lambda_{1}$ and $\lambda_{2}$ such that eq. (3) is satisfied for a non-zero vector $\mathbf{u}$, and the determination of the vector $\mathbf{u}$ itself within a constant complex multiplier.

There are essentially two different approaches to the development of numerical schemes for this problem. In the first approach, the condition of a vanishing determinant for the existence of 
a non-zero vector $u$ is used to obtain the values of $\lambda_{1}$ and $\lambda_{2}$. The eigenvector $u$ is obtained independently afterwards. Thus,

$$
\operatorname{det}\left[A\left(\lambda_{1}, \lambda_{2}\right)\right]=D\left(\lambda_{1}, \lambda_{2}\right)=0
$$

Eq. (4) is one complex equation in two real unknowns, $\lambda_{1}$ and $\lambda_{2}$, and can be solved by any of a variety of quasi-Newton methods. Eq. (4) is first rewritten in terms of its real and imaginary parts as

$$
\begin{aligned}
& D_{R}\left(\lambda_{1}, \lambda_{2}\right)=0 \\
& D_{I}\left(\lambda_{1}, \lambda_{2}\right)=0
\end{aligned}
$$

Eqs. (5) represent two nonlinear equations in two unknowns, $\lambda_{1}$ and $\lambda_{2}$. The typical iteration scheme for their solution would then be

$$
\left\{\begin{array}{l}
\lambda_{1} \\
\lambda_{2}
\end{array}\right\}(k+1)=\left\{\begin{array}{l}
\lambda_{1} \\
\lambda_{2}
\end{array}\right\}(k)-\mathbf{J}_{(k)}^{-1}\left\{\begin{array}{l}
D_{R} \\
D_{I}
\end{array}\right\}(k)
$$

where $k$ is the iteration number and $\mathbf{J}$ is the Jacobian matrix of eq. (5) and is given by

$$
\mathbf{J}=\left[\begin{array}{ll}
\frac{\partial D_{R}}{\partial \dot{\lambda}_{1}} & \frac{\partial D_{R}}{\partial \dot{\lambda}_{2}} \\
\frac{\partial D_{I}}{\partial \lambda_{1}} & \frac{\partial D_{I}}{\partial \dot{\lambda}_{2}}
\end{array}\right]
$$

If the Jacobian is analytically evaluated, eq. (6) represents Newton's method. Quasi-Newton methods approximate the Jacobian in some manner. Hassig (1971) and Stark (1984), for example, have used this approach, solving eq. (4) by the Regula Falsi and the secant methods respectively.

In the second approach, proposed by Cardani and Mantegazza (1978), the eigenvalue problem of eq. (3) along with a normalizing condition on the eigenvector $u$ are treated as a set of nonlinear equations in the unknowns, $\mathbf{u}, \lambda_{1}$ and $\lambda_{2}$. These nonlinear equations are then solved using standard techniques, obtaining the complete eigentriple simultaneously. While this approach obviously results in a much larger system of non-linear equations than the previous determinant equation approach, it has recently been advocated by Meyer (1988) over the determinant equation 
approach because "the basic drawback to solving the determinant equation is that since it is impractical to compute derivatives of the determinant with respect to system parameters, only firstorder convergent methods such as Regula Falsi or a secant method can be used, instead of more rapidly-converging methods such as Newton's method " and "convergence to the wrong root, known as mode switching, can occur when two modes are nearly equal ". Meyer (1988) presented a continuation method for the solution of eq. (3) using the second approach.

This paper addresses the first drawback noted by Meyer (1988). A practical iterative scheme, for solving the determinant equation with a convergence rate that is more rapid than the secant method and approaching that of Neuton's method, is proposed. It is first shown that all the derivatives of the determinant can be evaluated at the cost of a single matrix inversion. Further, a quasi- Newton method, based on Broyden's updates for the derivatives of the matrix $\mathbf{A}$ (rather than for the Jacobian matrix as is done in Broyden's method), is shown to be more rapidly convergent by means of examples. The second drawback of converging to the wrong root will not be addressed in this paper. However, this is felt to be only a minor limitation because most practical acroelastic structures do not often give rise to nearly equal modes that flutter.

The eigenvector $\mathbf{u}$ (flutter mode, in acroelastic literature) and the left eigenvector $\mathbf{v}$ can be computed very efficiently using the information available at the time of convergence of the iterative scheme in an inverse iteration procedure.

\section{Computation of Eigenvalues}

We consider the computation of the eigenvalue-pairs for eq. (3) by solving eqs. (5) through the iterative scheme of eq. (6). Note that analytical evaluation of the Jacobian requires the derivatives of the characteristic determinant, $D$. The derivatives of the characteristic determinant are computed by using the following result, known as the Trace theorem, proved by Lancaster(1964):

If the elements of $\mathbf{A}(\alpha)$ are differentiable functions of $\alpha$, then for any $\alpha$ for which the characteristic determinant $D(x) \neq 0$,

$$
\frac{\partial D}{\partial \alpha}=D(\alpha) \cdot \text { trace of }\left(\mathbf{A}^{-1} \frac{\partial A}{\partial \alpha}\right)
$$


Using eqs. (7) and (8), the iterative scheme of eq. (6) can be written as

$$
\left\{\begin{array}{l}
\lambda_{1} \\
\lambda_{2}
\end{array}\right\}(k+1)=\left\{\begin{array}{l}
\lambda_{1} \\
\lambda_{2}
\end{array}\right\}(k)-\frac{1}{\left(g_{R} h_{I}-h_{R} g_{I}\right)_{(k)}}\left\{\begin{array}{c}
h_{I} \\
-g_{I}
\end{array}\right\}(k)
$$

where

$$
\begin{aligned}
& g_{R}+i g_{I}=\text { trace of }\left(\mathrm{A}^{-1} \frac{\partial \mathrm{A}}{\partial \lambda_{1}}\right) \\
& h_{R}+i h_{I}=\text { trace of }\left(\mathrm{A}^{-1} \frac{\partial \mathrm{A}}{\partial \dot{\lambda}_{2}}\right)
\end{aligned}
$$

Thus, the trace theorem gives us a practical method for calculating the derivatives of the determinant. Note that, in evaluating eq. (10), matrix multiplication need not be performed, since only the trace is required. The computational cost of the matrix inversion required in eqs. (10) is not significant if the use of such derivatives improves the convergence rate of the determinant equation solution. This is because the cost of inverting the matrix $\mathbf{A}$ is usually negligible in comparison to the cost of evaluating it. The iteration is terminated when $\lambda_{1}$ and $\lambda_{2}$ satisfy some convergence criteria or the matrix $A$ cannot be inverted.

In eq. (10), if the derivatives of the matrix $\mathbf{A}$ with respect to the eigenvalues $\lambda_{1}$ and $\lambda_{2}$ are evaluated analytically, then eq. (9) is equivalent to Neuton's method and thus gives quadratic convergence. Analytical differentiation of $\mathbf{A}$ is not often practical and one resorts to approximate evaluation of the derivatives of $\mathbf{A}$, sacrificing quadratic convergence and hence increasing the number of iterations required for convergence. Approximate derivatives of $\mathrm{A}$ can be evaluated simply by finite-differences. However, finite-differencing increases the computational cost of each iteration, further degrading the efficiency of the iterative procedure. It is therefore proposed that the derivatives of $\mathbf{A}$ be evaluated during the iteration using Broyden's update formula (Dennis and More, 1977), which is used in Broyden's method to update the Jacobian. Numerical examples shown later indicate that this updating scheme, in combination with eqs. (9) and (10), results in an iterative scheme with a high rate of convergence (close to that of Newton's method) with the same cose per iteration as that of secant and Broyden's methods. 
The elements of $A$ are assumed to be linear functions of $\lambda_{1}$ and $\lambda_{2}$ in the direction of the last iterative move. Hence, the derivatives of $A$ at the $k$-th iterative solution satisfy

$$
\mathbf{A}_{(k-1)}=\mathbf{A}_{(k)}+\left(\frac{\partial \mathbf{A}}{\partial \lambda_{1}}\right)_{(k)}\left(\lambda_{1(k-1)}-\lambda_{1(k)}\right)+\left(\frac{\partial \mathbf{A}}{\partial \lambda_{2}}\right)_{(k)}\left(\lambda_{2(k-1)}-\lambda_{2(k)}\right)
$$

In addition, the derivatives of $\mathbf{A}$ in the direction orthogonal to the last iterative move are assumed to be the same at the $k$-th and the $(k-1)$-th iterative solutions. This implies

$$
\begin{aligned}
\left(\frac{\partial \mathbf{A}}{\partial \lambda_{1}}\right)_{(k)}\left(\dot{\lambda}_{1}-\lambda_{1}(k)\right. & +\left(\frac{\partial \mathbf{A}}{\partial \dot{\lambda}_{2}}\right)_{(k)}\left(\lambda_{2}-\lambda_{2(k)}\right) \\
= & \left(\frac{\partial \mathbf{A}}{\partial \dot{\lambda}_{1}}\right)_{(k-1)}\left(\lambda_{1}-\lambda_{1(k)}\right)+\left(\frac{\partial \mathbf{A}}{\partial \lambda_{2}}\right)_{(k-1)}\left(\lambda_{2}-\lambda_{2(k)}\right)
\end{aligned}
$$

for any $\lambda_{1}$ and $\lambda_{2}$ satisfying

$$
\left(\lambda_{1}-\lambda_{1(k)}\right)\left(\lambda_{1(k-1)}-\lambda_{1(k)}\right)+\left(\lambda_{2}-\lambda_{2(k)}\right)\left(\lambda_{2(k-1)}-\lambda_{2(k)}\right)=0
$$

Eqs. (11)-(13) uniquely determine $\left(\frac{\partial \mathbf{A}}{\partial \dot{\lambda}_{1}}\right)_{(k)}$ and $\left(\frac{\partial \mathbf{A}}{\partial \dot{\lambda}_{2}}\right)_{(k)}$. Lising the notation $\delta \lambda_{1(k)}=\lambda_{1(k-1)}-\lambda_{1(k)}$ and $\delta \lambda_{2(k)}=\lambda_{2(k-1)}-\lambda_{2(k)}, \quad\left(\frac{\partial \mathbf{A}}{\partial \lambda_{1}}\right)(k)$ is obtained as

$$
\left(\frac{\partial \mathbf{A}}{\partial \dot{\lambda}_{1}}\right)_{(k)}=\frac{\left(\mathbf{A}_{(k-1)}-\mathbf{A}_{(k)}\right) \delta \dot{\lambda}_{1(k)}+\left[\left(\frac{\partial \mathbf{A}}{\partial \dot{\lambda}_{1}}\right)_{(k-1)} \delta \dot{\lambda}_{2(k)}-\left(\frac{\partial \mathbf{A}}{\partial \lambda_{2}}\right)(k-1) \delta \dot{\lambda}_{1(k)}\right] \delta \lambda_{2(k)}}{\delta \dot{\lambda}_{1(k)}^{2}+\delta \dot{\lambda}_{2(k)}^{2}}
$$

A similar expression can be obtained for $\left(\frac{\partial A}{\partial \dot{\lambda}_{2}}\right)_{(k)}$ by simply exchanging the subscripts 1 and 2 for $\lambda$ in eq. (14).

\section{Computation of Eigenvectors}

The eigenvector $\mathbf{u}$ (flutter mode, in aeroelastic literature) can be computed very efficiently using the information available at the time of convergence of the iterative scheme, eq. (9), in an inverse iter- 
ation procedure. If $\mathbf{u}_{(0)}$ is any vector that is not totally deficient in the eigenvector, the following inverse iteration scheme can be used to calculate the eigenvector.

$$
\mathbf{u}_{(k)}=\mathbf{A}^{-1} \mathbf{u}_{(k-1)} \quad k=1,2, \ldots
$$

At the time of convergence of the iteration given by eq. (9), the matrix $\mathrm{A}^{-1}$ is already available in decomposed form and can be used directly in eq. (15).

Sensitivity calculations sometimes also need the left eigenvector associated with $\lambda_{1}$ and $\lambda_{2}$. The left eigenvector is defined by the equation

$$
\mathbf{v}^{T} \mathbf{A}\left(\hat{\lambda}_{1}, \lambda_{2}\right)=\mathbf{0}^{T}
$$

Once again, inverse iteration can be used to calculate the left eigenvector as follows. If $\mathbf{v}_{(0)}$ is any vector that is not totally deficient in the left eigenvector,

$$
v_{(k)}=\left[A^{T}\right]^{-1} v_{(k-1)} \quad k=1,2, \ldots
$$

As in the case of the linear eigenvalue problem, one step of the inverse iteration scheme is normally sufficient to obtain the eigenvector within a reasonable accuracy. There is again no need for another decomposition to solve eq. (17), as $\left[\mathbf{A}^{T}\right]^{-1}=\left[\mathbf{A}^{-1}\right]^{T}$ and the previous decomposition can be used by replacing backward substitution with forward substitution.

\section{Numerical Examples}

The performance of the above algorithms is demonstrated through two numerical examples. Both examples present complex transcendental eigenvalue problems having known pairs of real eigenvalues. Example 1 uses trigonometric functions and example 2 uses hyperbolic functions. The examples were designed to illustrate the various features of the algorithms and for easy verification of the results. 
Example 1:For this example, the complex transcendental matrix is given by

$$
\mathbf{A}\left(\lambda_{1}, \lambda_{2}\right)=\left[\begin{array}{ll}
z_{1} & \sin \left(\beta_{1} \lambda_{2}+\beta_{2}\right) \\
z_{2} & \sin \left(\alpha_{1} \lambda_{1}+\alpha_{2}\right)
\end{array}\right]
$$

This eigenproblem has an infinite number of pairs of real eigenvalues. A family of such pairs is given by

$$
\begin{aligned}
& \lambda_{1}=\frac{r \pi-\alpha_{2}}{\alpha_{1}} \quad \lambda_{2}=\frac{s \pi-\beta_{2}}{\beta_{1}} \\
& r=0, \pm 1, \pm 2, \ldots \quad s=0, \pm 1, \pm 2, \ldots
\end{aligned}
$$

The nominal design point is represented by the values

$$
\begin{array}{ll}
z_{1}=1+i & z_{2}=1-i \\
\alpha_{1}=2 & \alpha_{2}=-1 \\
\beta_{1}=2 & \beta_{2}=-3
\end{array}
$$

The eigenvalue pair of interest is given by $\lambda_{1}=0.5$ and $\lambda_{2}=1.5$.

Example 2:In the second example, the eigenproblem involves the complex transcendental matrix $\mathbf{A}\left(\lambda_{1}, \lambda_{2}\right)$, the elements of which are given by

$$
\begin{aligned}
& a_{p q}=z_{p q}^{(1)} \sinh \left(x_{p} \lambda_{1}+\beta_{p}\right)+z_{p q}^{(2)} \sinh \left(\alpha_{p}^{2} \lambda_{2}+\delta_{p}\right) \\
& p=1,2, \ldots, n \quad q=1,2, \ldots, n
\end{aligned}
$$

$z_{p q}^{(1)}$ and $z_{p q}^{(2)}$ represent complex constants chosen randomly for this example. $\alpha_{p}, \beta_{p}$ and $\delta_{p}$ are real quantities that serve as design parameters. It can easily be shown that when the elements of the matrix $\mathbf{A}$ are given by eq. (21), the eigenproblem of eq. (3) has at least $n$ pairs of real eigenvalues that are independent of the constants $z_{p q}^{(1)}$ and $z_{p q}^{(2)}$. These are given by

$$
\lambda_{1}=-\frac{\beta_{p}}{\alpha_{p}}, \quad \lambda_{2}=-\frac{\delta_{p}}{\alpha_{p}^{2}}, \quad p=1,2, \ldots, n
$$


There are additional pairs of real eigenvalues that depend on $z_{p q}^{(1)}$ and $z_{p q}^{(2)}$.

The results presented here for example 2 are for $n=6$ and the nominal design point is represented by the values

$$
\begin{aligned}
& \alpha_{p}=p \\
& \beta_{p}=-0.05 p^{2} \\
& \delta_{p}=-0.005 p^{3} \\
& \neq 2 \text { and } \\
& \alpha_{2}=1 \\
& \beta_{2}=-0.5 \\
& \delta_{2}=-0.1
\end{aligned}
$$

The eigenvalue pair of interest is given by $\lambda_{1}=0.5$ and $\lambda_{2}=0.1$.

\section{Rate of convergence}

The rate of convergence of the present solution procedure was earlier shown by Murthy and Kaza (1988) to be more rapid than that of the generalized secant method for the case of the aeroelastic analysis of propfan blades. In this paper, further results are presented comparing the present solution procedure, represented by the iterative scheme of eq. (9), to Broyden's method and Newton's method. In Broyden's method, the directional update is applied to the Jacobian matrix. The present procedure differs from Broyden's method in that the directional update is applied to the system matrix rather than the Jacobian matrix. The eigenproblems associated with both the above examples are solved by Broyden's method, the present procedure and Newton's method. For Broyden's method and the present procedure, the exact analytical derivatives were used to calculate the initial Jacobian matrix. For Broyden's method and the present procedure, only the matrix $\mathbf{A}$ has to be evaluated once per iteration. For Neuton's method, the matrix $\mathbf{A}$ and its analytical de- 
rivatives with respect to $\lambda_{1}$ and $\lambda_{2}$ are evaluated once per iteration. The same convergence criterion was used for all results. Tables 1 and 2 show, for the eigenproblems posed by cxamples 1 and 2 respectively, the number of iterations required for convergence starting with various initial guesses. The tables demonstrate that the present procedure is far superior to Broyden's method in terms of the number of iterations required for convergence. Comparison to Newton's method also demonstrates that at most two more iterations are required with the present procedure than with Newton's method. Thus, the present solution procedure appears to have a rate of convergence that is close to that of the quadratically convergent Newton's method.

\section{Sensitivity Analysis}

Let the matrix $\mathbf{A}$ and hence the eigentriple $\lambda_{1}, \lambda_{2}$ and $\mathbf{u}$ be functions of design parameter vector $\mathbf{p}$ with individual parameters denoted by Greek subscripts, e.g. $p_{\alpha}$. We assume that the design parameters are all real and that the pair $\lambda_{1}$ and $\lambda_{2}$ is a distinct solution for eq. (4).

\section{Eigenvalue Sensitivities}

The objective is to obtain expressions for the eigenvalue sensitivities, $\frac{\partial \lambda_{1}}{\partial p_{\alpha}}$ and $\frac{\partial \lambda_{2}}{\partial p_{\alpha}}$. Differentiating eq. (4) with respect to the design parameter $p_{\alpha}$, we have,

$$
\frac{\partial D}{\partial p_{\alpha}}+\frac{\partial D}{\partial \lambda_{1}} \frac{\partial \dot{\lambda}_{1}}{\partial p_{\alpha}}+\frac{\partial D}{\partial \lambda_{2}} \frac{\partial \dot{\lambda}_{2}}{\partial p_{\alpha}}=0
$$

Eq. (25), once again involves the derivatives of the determinant of $\mathbf{A}$, this time evaluated at the solution obtained at the convergence of the iterative scheme given by eq. (9). However, the trace theorem in the form of eq. (8) cannot be used to calculate these derivatives, as the the determinant vanishes at the solution $\lambda_{1}$ and $\lambda_{2}$. Hence, eq. (8) is rewritten as

$$
\frac{\partial D}{\partial \alpha}=\text { trace of }\left(\operatorname{adj} \mathrm{A} \frac{\partial \mathbf{A}}{\partial \alpha}\right)
$$

using the relation between the inverse and the adjoint matrices, 


$$
\mathbf{A}^{-1}=\frac{\operatorname{adj} \mathbf{A}}{D}
$$

Direct calculation of the adjoint matrix for use in eq. (26) is computationally expensive and can be avoided as shown below.

Frazer, Duncan and Collar (1960) have shown that, if $\mathbf{A}(\lambda)$ is a $\lambda$-matrix and $\lambda_{0}$ is a simple root of the characteristic equation $\operatorname{det} \mathbf{A}(\lambda)=0$, then the rows and columns of the adjoint matrix, $\operatorname{adj} \mathbf{A}\left(\lambda_{0}\right)$, are directly proportional respectively to the left and right eigenvectors corresponding to 2. It can similarly be shown that the adjoint matrix has the same property in the case of the transcendental eigenprobem with real eigenvalues, given by eq. (3), if the left eigenvector is defined by eq. (16). Thus, if $\lambda_{1}$ and $\lambda_{2}$ form a simple root pair of eq. (4) and $\mathbf{u}$ and $\mathbf{v}$ satisfy eq. (3) and eq. (16) respectively, then $\operatorname{adj} \mathbf{A}\left(\lambda_{1}, \lambda_{2}\right)$ has rows and columns directly proportional to $\mathbf{v}$ and $\mathbf{u}$ respectively. That is,

$$
\operatorname{adj} \mathbf{A}\left(\dot{\lambda}_{1}, \dot{\lambda}_{2}\right)=d \mathbf{u} \mathbf{v}^{T}
$$

where $d$ is some non-zero constant dependent on the eigenvector normalization. Thus, the derivatives of the determinant at the solution, $\lambda_{1}$ and $\lambda_{2}$, can be expressed as

$$
\frac{\partial D}{\partial \alpha}=d \text { trace of }\left(\mathbf{u r}^{T} \frac{\partial \mathbf{A}}{\partial \alpha}\right)
$$

Or,

$$
\frac{\partial D}{\partial \alpha}=d \mathbf{v}^{T} \frac{\partial \mathbf{A}}{\partial \alpha} \mathbf{u}
$$

Using eq. (29) to evaluate the derivatives of the determinant in eq. (25) and cancelling out the unknown constant $d$, we obtain the sensitivity equation

$$
\mathbf{v}^{T} \frac{\partial \mathbf{A}}{\partial p_{\alpha}} \mathbf{u}+\mathbf{v}^{T} \frac{\partial \mathbf{A}}{\partial \lambda_{1}} \mathbf{u} \frac{\partial \dot{\lambda}_{1}}{\partial p_{\alpha}}+\mathbf{v}^{T} \frac{\partial \mathbf{A}}{\partial \lambda_{2}} \mathbf{u} \frac{\partial \lambda_{2}}{\partial p_{\alpha}}=0
$$

Since the eigenvalues $\lambda_{1}$ and $\lambda_{2}$ are restricted to real values and the design parameters are assumed to be real, the sensitivities of the eigenvalues, $\frac{\partial \lambda_{1}}{\partial p_{\alpha}}$ and $\frac{\partial \lambda_{2}}{\partial p_{\alpha}}$, must also be real. Thus, eq. (30) 
is a complex equation, in the two real unknowns, $\frac{\partial \lambda_{1}}{\partial p_{\alpha}}$ and $\frac{\partial \lambda_{2}}{\partial p_{\alpha}}$. Unlike eq. (4), the sensitivity equation is linear and it can be solved exactly. Sobieski(1986) pointed out that the sensitivity equation is always linear, even for nonlinear systems. Solution of eq. (30) gives us the following expressions for the eigenvalue sensitivities

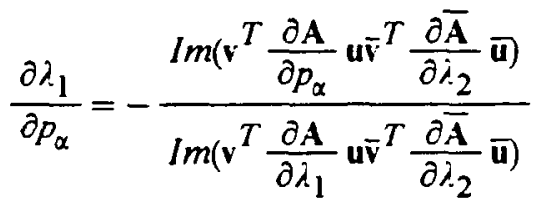

$$
\begin{aligned}
& \frac{\partial \lambda_{2}}{\partial p_{\alpha}}=-\frac{\operatorname{Im}\left(\mathbf{v}^{T} \frac{\partial \mathbf{A}}{\partial p_{\alpha}} \mathbf{u}^{T} \frac{\partial \overline{\mathbf{A}}}{\partial \dot{\lambda}_{1}} \overline{\mathbf{u}}\right)}{\operatorname{Im}\left(\mathbf{v}^{T} \frac{\partial \mathbf{A}}{\partial \dot{\lambda}_{2}} \mathbf{u v}^{T} \frac{\partial \overline{\mathbf{A}}}{\partial \dot{\lambda}_{1}} \overline{\mathbf{u}}\right)}
\end{aligned}
$$

where the bar indicates the complex conjugate. These expressions are essentially the discrete equivalents of the flutter point sensitivities developed by Seyranian (1982) for the case of a slender elastic beam in incompressible flow. Note that, while the eigenvectors are only determined up to an arbitrary complex multiplier in the absence of appropriate normalizing conditions, the eigenvalue sensitivities are independent of the normalizing condition.

\section{Integrated Computation of Eigenvalue and Eigenvector Sensitivities}

Murthy and Haftka (1988) described two computational approaches, the Direct approach and the Adjoint approach, for calculating the sensitivities of the eigenvalues and eigenvectors of linear algebraic eigenvalue problems and showed that both the approaches are competitive, under different sets of conditions. The Adjoint approach requires all the left and the right eigenvectors and the Direct approach requires no left eigenvectors. The lack of bi-orthogonality relationships in the case of the eigenvalue problem of eq. (3) eliminates the possibility of utilizing the Adjoint approach for calculating derivatives of the eigenvectors, because the bi-orthogonality property is crucial for such an approach.

When eigenvector sensitivities are required, both the eigenvalue and the eigenvector sensitivities can be computed in an integrated manner, using the Direct approach. Bindolino and 
Mantegazza (1987) presented one such method, which operates in the real domain rather than the complex domain. The normalization condition they used for the eigenvectors is unreliable when the eigenvectors are complex. Murthy and Haftka (1988) discussed the merits of different normalization conditions to be used in sensitivity analysis computations. In the following, a Direct approach, requiring no left eigenvectors and based on proper eigenvector normalization, is developed for the integrated computation of the eigenvalue and eigenvector sensitivities for the eigenproblem posed by eq. (3).

Direct differentiation of eq. (3) with respect to the parameter $p_{\alpha}$ gives

$$
\frac{\partial \mathbf{A}}{\partial p_{\alpha}} \mathbf{u}+\frac{\partial \mathbf{A}}{\partial \lambda_{1}} \mathbf{u} \frac{\partial \lambda_{1}}{\partial p_{\alpha}}+\frac{\partial \mathbf{A}}{\partial \dot{\lambda}_{2}} \mathbf{u} \frac{\partial \lambda_{2}}{\partial p_{\alpha}}+\mathbf{A} \frac{\partial \mathbf{u}}{\partial p_{\alpha}}=0
$$

which can be written in matrix form as

$$
\left[\begin{array}{lll}
\mathbf{A} & \frac{\partial \boldsymbol{A}}{\partial \dot{\lambda}_{1}} \mathbf{u} & \frac{\partial \boldsymbol{A}}{\partial \dot{i}_{2}} \mathbf{u}
\end{array}\right]\left\{\begin{array}{l}
\frac{\partial \mathbf{u}}{\partial p_{\alpha}} \\
\frac{\partial \dot{\lambda}_{1}}{\partial p_{\alpha}} \\
\frac{\partial \dot{j}_{2}}{\partial p_{\alpha}}
\end{array}\right\}=-\frac{\partial \mathbf{A}}{\partial p_{\alpha}} \mathbf{u}
$$

Eq. (34) consists of $n$ linear equations in $(n+2)$ unknowns, which are the $n$ components of $\frac{\partial \mathbf{u}}{\partial p_{\alpha}}$ and $\frac{\partial \lambda_{1}}{\partial p_{\alpha}}$ and $\frac{\partial \dot{\lambda}_{2}}{\partial p_{\alpha}}$. Thus, two additional conditions must be stipulated in order to obtain a solution of eq. (34). One of these is obtained by normalizing the eigenvector to make it unique and the other by restricting $\frac{\partial \lambda_{1}}{\partial p_{\alpha}}$ and $\frac{\partial \lambda_{2}}{\partial p_{\alpha}}$ to real values.

The first condition is easily enforced by normalizing the eigenvector so that a non-zero element is unity. Let $u_{m}$ be such an element. Then, since $u_{m}=1$,

$$
\frac{\partial u_{m}}{\partial p_{\alpha}}=0
$$

and the $m$-th column of the coefficient matrix in eq. (34) can be deleted. Eq. (35) also reduces the number of unknowns in eq. (34) by one. 
The second condition is best enforced by partitioning eq. (34) as follows. Writing the $l$-th equation of the system given by eq. (34) separately, we have

$$
\mathbf{C} \frac{\partial \mathbf{x}}{\partial p_{\alpha}}+\mathbf{n}_{1} \frac{\partial \lambda_{1}}{\partial p_{\alpha}}+\mathbf{n}_{2} \frac{\partial \lambda_{2}}{\partial p_{\alpha}}=-\mathbf{n}_{\alpha}
$$

and

$$
\mathbf{a}_{l}^{T} \frac{\partial \mathbf{x}}{\partial p_{\alpha}}+\eta_{1} \frac{\partial \lambda_{1}}{\partial p_{\alpha}}+\eta_{2} \frac{\partial \lambda_{2}}{\partial p_{\alpha}}=-\eta_{\alpha}
$$

where

$$
\begin{aligned}
& \mathbf{C}=\mathbf{A}_{l-\text { th row and } m \text {-th column deleted }} \\
& \mathbf{x}=\mathbf{u}_{m-\text { th } \text { element deleted }} \\
& \mathbf{n}_{1}=\left\{\frac{\partial \mathbf{A}}{\partial \lambda_{1}} \mathbf{u}\right\}_{l-\text { th element deleted }} \\
& \mathbf{n}_{2}=\left\{\frac{\partial \mathbf{A}}{\partial \lambda_{2}} \mathbf{u}\right\}_{l-\text { th element deleted }} \\
& \mathbf{n}_{\alpha}=\left\{\frac{\partial \mathbf{A}}{\partial p_{\alpha}} \mathbf{u}\right\}_{l-\text { th } \text { element deleted }} \\
& \mathbf{2}_{l}^{T}=l-\text { th row of } \mathbf{A} \text { with } m-\text { th column deleted } \\
& \eta_{1}=l-\text { th element of }\left\{\frac{\partial \mathbf{A}}{\partial \lambda_{1}} \mathbf{u}\right\} \\
& \eta_{\alpha}=l-\text { th element of }\left\{\frac{\partial \mathbf{A}}{\partial p_{\alpha}} \mathbf{u}\right\} \\
& \eta_{2}=l-\text { th element of }\left\{\frac{\partial \mathbf{A}}{\partial \lambda_{2}} \mathbf{u}\right\}
\end{aligned}
$$

Eliminating $\frac{\partial \mathbf{x}}{\partial p_{\alpha}}$ from eqs. (36) and (37), we have 


$$
\left(\eta_{1}-\mathbf{b}_{l}^{T} \mathbf{n}_{1}\right) \frac{\partial \lambda_{1}}{\partial p_{\alpha}}+\left(\eta_{2}-\mathbf{b}_{l}^{T} \mathbf{n}_{2}\right) \frac{\partial \lambda_{2}}{\partial p_{\alpha}}=-\left(\eta_{\alpha}-\mathbf{b}_{l}^{T} \mathbf{n}_{\alpha}\right)
$$

where

$$
\mathbf{b}_{l}=\left[\mathbf{C}^{T}\right]^{-1} \mathbf{a}_{l}
$$

The condition of real eigenvalue sensitivities is now enforced on eq. (38) to obtain

$$
\begin{aligned}
& \frac{\partial \lambda_{1}}{\partial p_{\alpha}}=-\frac{\operatorname{Im}\left[\left(\eta_{\alpha}-\mathbf{b}_{l}^{T} \mathbf{n}_{\alpha}\right)\left(\bar{\eta}_{2}-\overline{\mathbf{b}}_{l}^{T} \overline{\mathbf{n}}_{2}\right)\right]}{\operatorname{Im}\left[\left(\eta_{1}-\mathbf{b}_{l}^{T} \mathbf{n}_{1}\right)\left(\bar{\eta}_{2}-\overline{\mathbf{b}}_{l}^{T} \overline{\mathbf{n}}_{2}\right)\right]} \\
& \frac{\partial \lambda_{2}}{\partial \rho_{\alpha}}=-\frac{\operatorname{Im}\left[\left(\eta_{\alpha}-\mathbf{b}_{l}^{T} \mathbf{n}_{\alpha}\right)\left(\bar{\eta}_{1}-\overline{\mathbf{b}}_{l}^{T} \overline{\mathbf{n}}_{1}\right)\right]}{\operatorname{Im}\left[\left(\eta_{2}-\mathbf{b}_{l}^{T} \mathbf{n}_{2}\right)\left(\bar{\eta}_{1}-\overline{\mathbf{b}}_{l}^{T} \overline{\mathbf{n}}_{1}\right)\right]}
\end{aligned}
$$

Thus, the following procedure can be used to obtain the derivatives $\frac{\partial \mathbf{u}}{\partial p_{\alpha}}, \frac{\partial \lambda_{1}}{\partial p_{\alpha}}$ and $\frac{\partial \lambda_{2}}{\partial p_{\alpha}}$.

1. Normalize the eigenvector such that $u_{m}=1$.

2. Form the LU decomposition of the matrix $\mathbf{C}$.

3. Solve $\mathbf{b}_{l}=\left[\mathbf{C}^{T}\right]^{-1} \mathbf{a}_{l}$ by forward substitution.

4. Calculate $\frac{\partial \dot{\lambda}_{1}}{\partial p_{x}}$ and $\frac{\partial \dot{\lambda}_{2}}{\partial p_{\alpha}}$ from eqs. (39) and (40).

5. Calculate $\frac{\partial \mathbf{x}}{\partial p_{x}}$ from eq. (36) by backward substitution.

6. Expand $\frac{\partial \mathbf{x}}{\partial p_{\alpha}}$ to $\frac{\partial \mathbf{u}}{\partial p_{\alpha}}$ by setting $\frac{\partial u_{m}}{\partial p_{\alpha}}=0$

The proper choice of the two indices $l$ and $m$ is of great importance for the above procedure to calculate accurate sensitivities. The indices must be chosen so that the resulting matrix $\mathbf{C}$ is well-conditioned. Note that $\mathrm{C}$ is obtained from the singular matrix $A\left(\lambda_{1}, \lambda_{2}\right)$ by deleting the row corresponding to the index $l$ and the column corresponding to the index $m$. Hence, in the absence of multiple solutions, for the matrix $\mathbf{C}$ to be non-singular, the $l$-th row and the $m$-th column of $A$ 
must be linearly dependent on the other rows and the other columns respectively. In other words, $\mathrm{C}$ is non-singular if and only if $v_{l} \neq 0$ and $u_{m} \neq 0$. (For a detailed discussion of this point, see Nelson, 1976). Therefore, to obtain a well-conditioned matrix $\mathbf{C}$, a reasonable choice of the indices $l$ and $m$ would be such that $v_{l}$ is the element of largest absolute value in $\mathbf{v}$ and $u_{m}$ is the element of largest absolute value in $\mathbf{u}$. Thus, while the direct method does not require the left eigenvector in principle, it may still be necessary to calculate the left eigenvector in order to choose the index l that results in a well-conditioned matrix $\mathbf{C}$.

\section{Finite Difference Sensitivity Analysis}

The eigenvalue and eigenvector sensitivities can also be calculated by finite differences. While the finite difference approach has the virtues of simplicity and ease of implementation, it is known to suffer from efficiency and accuracy problems. For example, the finite difference approach requires the solution of the nonlinear equation, eq. (3), once for each design variable. On the other hand, the analytical approach requires the solution of only a linear equation, eq. (30) or (33), with the same coefficient matrix for all design variables. However, Haftka (1985) has pointed out that, for iteratively solved problems, the finite difference methods are usually superior to the analytical methods in terms of efficiency, as the solution at the nominal point is usually a very good initial guess for the solution at the perturbed point.

The accuracy problems still remain, because of the truncation and the round-off errors inherent to the finite difference approach. For iteratively solved problems, these difficulties are exacerbated due to the presence of errors arising from the early termination of the iterative process and the different initial guesses used for the nominal and the perturbed solutions. Haftka (1985) presented a novel technique for controlling the magnitude of the errors associated with iteratively solved problems. More accurate derivatives are obtained by treating the converged solution as the exact solution of an approximate equation instead of the approximate solution of an exact equation. The finite difference approach is not considered in this paper but is mentioned here to alert the reader to an important altemative to the analytical approach. 


\section{Numerical Examples}

A computer program was developed that implements both of the approaches to the sensitivity analysis. The above algorithms were applied to the two numerical examples considered earlier. Both examples present complex transcendental eigenvalue problems having known real valued eigenvalues that are dependent on certain parameters in a known manner.

Example 3:For this example, sensitivity analysis of the eigenproblem presented in example 1, with respect to the parameters $\alpha_{1}$ and $\beta_{1}$, is considered. The analytical sensitivities of the family of eigenvalue-pairs given by eq. (19) can be expressed as

$$
\begin{aligned}
& \frac{\partial \lambda_{1}}{\partial \alpha_{1}}=-\frac{r \pi-\alpha_{2}}{\alpha_{1}^{2}} \quad \frac{\partial \lambda_{2}}{\partial \alpha_{1}}=0 \\
& \frac{\partial \lambda_{1}}{\partial \beta_{1}}=0 \quad \frac{\partial \lambda_{2}}{\partial \beta_{1}}=-\frac{s \pi-\beta_{2}}{\beta_{1}^{2}} \\
& r=0, \pm 1, \pm 2, \ldots \quad s=0, \pm 1, \pm 2, \ldots
\end{aligned}
$$

Example 4:For this example, the eigenproblem is the one described in example 2 and the design parameters considered are $\alpha_{p}, \beta_{p}$ and $\delta_{p}$. The sensitivities of the eigenvalues given by eq. (22) can analytically be expressed as

$$
\begin{aligned}
& \frac{\partial \lambda_{1}}{\partial \alpha_{p}}=\frac{\beta_{p}}{\alpha_{p}^{2}} \quad \frac{\partial \lambda_{2}}{\partial \alpha_{p}}=\frac{2 \delta_{p}}{\alpha_{p}^{3}} \\
& \frac{\partial \lambda_{1}}{\partial \beta_{p}}=-\frac{1}{\alpha_{p}} \quad \frac{\partial \lambda_{2}}{\partial \beta_{p}}=0 \\
& \frac{\partial \lambda_{1}}{\partial \delta_{p}}=0 \quad \frac{\partial \lambda_{2}}{\partial \delta_{p}}=-\frac{1}{\alpha_{p}^{2}}
\end{aligned}
$$

The algorithms presented above correctly calculated the sensitivities of the pairs of real eigenvalues for the example problems. 


\section{Inaccuracy of the derivatives calculated by eq. (14)}

Eq. (31) and (32) require the derivatives of the matrix $\mathbf{A}$ with respect to the design parameter of interest, $p_{\alpha}$, and the two eigenvalues, $\lambda_{1}$ and $\lambda_{2}$. It may be expected that approximations to the derivatives of $A$ with respect to $\lambda_{1}$ and $\lambda_{2}$, which are available from eqs. (14) at the convergence of the iterative scheme of eq. (8), can be used in eqs. (31) and (32). However, this is not advisable because these approximations do not necessarily converge to exact values even though the corrections in the iterative scheme converge to Newton corrections. This behavior is demonstrated in Table 3 for typical elements of the system matrix of example 1. Table 3 demonstrates that the derivatives of $A$ at the convergence of the iterative scheme of eq. (9) deviate significantly from exact values, unless the initial guess is extremely good. Large deviations were observed in the case of example 2 also. As pointed out by Dennis and More (1977), this behavior is not unexpected for certain quasi-Newton methods including Broyden's method. Hence, it is recommended that the derivatives of $\mathrm{A}$ be re-evaluated at the solution for use in sensitivity analysis.

\section{Concluding Remarks}

In this paper, the complex transcendental eigenproblem with pairs of real eigenvalues is considered. An improved computational method for obtaining the eigenvalues of this eigenproblem is presented. The method is based on determinant iteration and achieves a high rate of convergence that is close to that of Newton's method with the same cost per iteration as the generalized secant and Broyden's methods. The method utilizes the philosophy of Broyden's updates, except that the updates are applied to the system matrix derivatives rather than the Jacobian matrix as in Broyden's method. Computational algorithms are also presented for calculating the sensitivities of these eigenvalues and the corresponding eigenvectors with respect to design parameters. One algorithm computes the sensitivities of the eigenvalues only, while the second algorithm computes the sensitivities of the eigenvalues as well as the eigenvectors in an integrated manner. The algorithms presented are verified by applying them to example problems. These algorithms are expected to prove useful in aeroelastic sensitivity analysis and optimization procedures. 


\section{Acknowledgment}

The author would like to acknowledge the many illuminating discussions with the late Dr. Krishna

Rao V. Kaza of NASA Lewis Research Center during the preparation of this paper.

\section{References}

Bindolino, G. and Mantegazza, P., 1987, "Aeroelastic Derivatives as a Sensitivity Analysis of Nonlinear Equations", AIAA Journal, Vol. 25, No. 8, pp. 1145-1146.

Bisplinghoff, R. L., Ashley, H. and Halfman, R. L., 1955, Aeroelasticity, Addison-Wesley, pp. 564-568.

Cardani, C. and Mantegazza, P., 1978, "Continuation and Direct Solution of the Flutter Equation", Computers and Structures, Vol. 8, No. 2, pp. 185-192.

Dennis, J. E. Jr. and More, J. J., 1977, "Quasi-Newton Methods, Motivation and Theory", SIA.M Review, Vol. 19 , No. 1, pp.46-89.

Frazer, R. A., Duncan, W. J. and Collar, 1960, Elementary Matrices, Cambridge University Press, Cambridge.

Frazer, R. A., 1946, "Bi-variate Partial Fractions and Their Applications to Flutter and Stability Problems", Proceedings of the Royal Society of London A, Vol. 185, pp. 465-484.

Fritzen, C. P. and Xordmann, R., 1982, "Influence of Parameter Changes to Stability Behavior of Rotors", Rotordynamic Instability Problems in High-Performance Turbomachinery, NASA CP.2250, pp. 284-306.

Haftka, R. T., 1985, "Sensitivity Calculations for Iteratively Solved Problems", International Journal for Numerical Methods in Engineering, Vol. 21, pp. 1535-1546.

Hassig, H. J., 1971, "An Approximate True Damping Solution of the Flutter Equation by Determinant Iteration", Joumal of Aircraft, Vol. 8, No. 11, pp. 885-889.

Lancaster, P., 1964, "Algorithms for Lambda-Matrices", Numerische Mathematik, Vol. 6, pp. 388-394.

Meyer, E. E., 1988, "Application of a New Continuation Method to Flutter Equations", Proceedings of the 29th Structures, Structural Dynamics and Materials Conference, Part 3, Williamsburg, VA, pp. 1118-1123.

Murthy, D. V. and Kaza, K. R. V., 1988, "A Computational Procedure for Automated Flutter Analysis", Communications in Applied Numerical Methods, (to appear).

Murthy, D. V. and Haftka, R. T., 1988, "Derivatives of Eigenvalues and Eigenvectors of a General Complex Matrix", International Joumal for Numerical Methods in Engineering, Vol. 26, No. 2, pp. 293-311.

Nelson, R. B., 1976, "Simplified Calculation of Eigenvector Derivatives", AlAA Journal, Vol. 14, o. 9, pp. 1201-1205.

Rokne, J., 1985, "Including Iterations for the Lambda-Matrix Eigenproblem", Computing, Vol. 35, pp. 207-218.

Seyranian, A. P., 1982, "Sensitivity Analysis and Optimization of Aeroelastic Stability", International Journal of Solids and Structures, Vol. 18, No. 9, pp. 791-807. 
Sobieszczanski-Sobieski, J., 1986, "The Case for Aerodynamic Sensitivity Analysis", Sensitivity Analysis in Engineering, NASA CP-2457, pp. 77-96.

Stark, V. J. E., 1984, "A Flutter Eigenvalue Program Based on the Newton-Raphson Method", AIAA Journal, Vol. 22, No. 7, pp. 993-995.

Taylor, D. L. and Kane, T. R., 1975, "Multiparameter Quadratic Eigenproblems", Joumal of Applied Mechanics, Vol. 42, pp. 478-483.

Wilkinson, J. H., 1965, The Algebraic Eigenvalue Problem, Clarendon Press, Oxford.

Table 1. - COMPARISON OF RATE OF CONVERGENCE - Example 1.

( Eigenvalue-pair of interest is $\lambda_{1}=0.5$ and $\lambda_{2}=1.5$ )

\begin{tabular}{|c|c|c|c|c|}
\hline \multicolumn{2}{|c|}{ Initial guess } & \multicolumn{3}{c|}{ Number of Iterations for Convergence } \\
\hline$\lambda_{1}$ & $\lambda_{2}$ & $\begin{array}{c}\text { Newton's } \\
\text { method }\end{array}$ & $\begin{array}{c}\text { Present } \\
\text { method }\end{array}$ & $\begin{array}{c}\text { Broyden's } \\
\text { method }\end{array}$ \\
\hline 0.4 & 1.5 & 3 & 4 & 4 \\
0.7 & 1.5 & 3 & 4 & 6 \\
0.3 & 1.3 & 3 & 5 & 6 \\
0.3 & 1.7 & 3 & 5 & 6 \\
0.7 & 1.3 & 3 & 5 & 6 \\
0.7 & 1.7 & 3 & 5 & 6 \\
0.9 & 1.9 & 4 & 6 & 10 \\
0.9 & 1.1 & 4 & 6 & 10 \\
0.4 & 1.9 & 4 & 5 & 13 \\
0.4 & 1.1 & 4 & 5 & 13 \\
\hline
\end{tabular}


Table 2. - COMPARISON OF RATE OF CONVERGENCE - Example 2.

( Eigenvalue-pair of interest is $\lambda_{1}=0.5$ and $\lambda_{2}=0.1$ )

\begin{tabular}{|c|c|c|c|c|}
\hline \multicolumn{2}{|c|}{ Initial guess } & \multicolumn{3}{|c|}{ Number of Iterations for Convergence } \\
\hline$\lambda_{1}$ & $\lambda_{2}$ & $\begin{array}{l}\text { Newton's } \\
\text { method }\end{array}$ & $\begin{array}{l}\text { Present } \\
\text { method }\end{array}$ & $\begin{array}{l}\text { Broyden's } \\
\text { method }\end{array}$ \\
\hline 0.51 & 0.11 & 5 & 5 & 19 \\
\hline 0.54 & 0.14 & 9 & 10 & $\begin{array}{l}\text { No Conver- } \\
\text { gence }\end{array}$ \\
\hline 0.60 & 0.20 & 14 & 20 & No Conver- \\
\hline 0.58 & 0.09 & 6 & 6 & 21 \\
\hline 0.49 & 0.11 & 5 & 5 & 14 \\
\hline 0.49 & 0.12 & 6 & 6 & $\begin{array}{c}38 \\
8\end{array}$ \\
\hline 0.40 & & & & \\
\hline
\end{tabular}

Table 3. - ACCLRACY OF MATRIX DERIVATIVES AT CONVERGENCE - Example 1. (Eigenvalue-pair of interest is $\lambda_{1}=0.5$ and $\lambda_{2}=1.5$ )

$$
\left(\frac{\partial a_{12}}{\partial \lambda_{1}}\right)_{\text {exact }}=0 . \quad\left(\frac{\partial a_{22}}{\partial i_{1}}\right)_{\text {exact }}=2 .
$$

\begin{tabular}{|l|c|c|c|}
\hline \multicolumn{2}{|c|}{ Initial guess } & \multicolumn{2}{c|}{ Derivative at Convergence } \\
\hline$\lambda_{1}$ & $\lambda_{2}$ & $\partial a_{12} / \partial \lambda_{1}$ & $\partial a_{22} / \partial \lambda_{1}$ \\
\hline 0.4 & 1.5 & 0.000 & 2.000 \\
0.7 & 1.5 & 0.000 & 2.000 \\
0.3 & 1.3 & 0.104 & 1.947 \\
0.3 & 1.7 & -0.104 & 1.947 \\
0.7 & 1.3 & -0.104 & 1.947 \\
0.7 & 1.7 & 0.104 & 1.947 \\
0.9 & 1.9 & 0.404 & 1.797 \\
0.9 & 1.1 & -0.404 & 1.797 \\
0.4 & 1.9 & -0.085 & 1.961 \\
0.4 & 1.1 & 0.085 & 1.961 \\
\hline
\end{tabular}




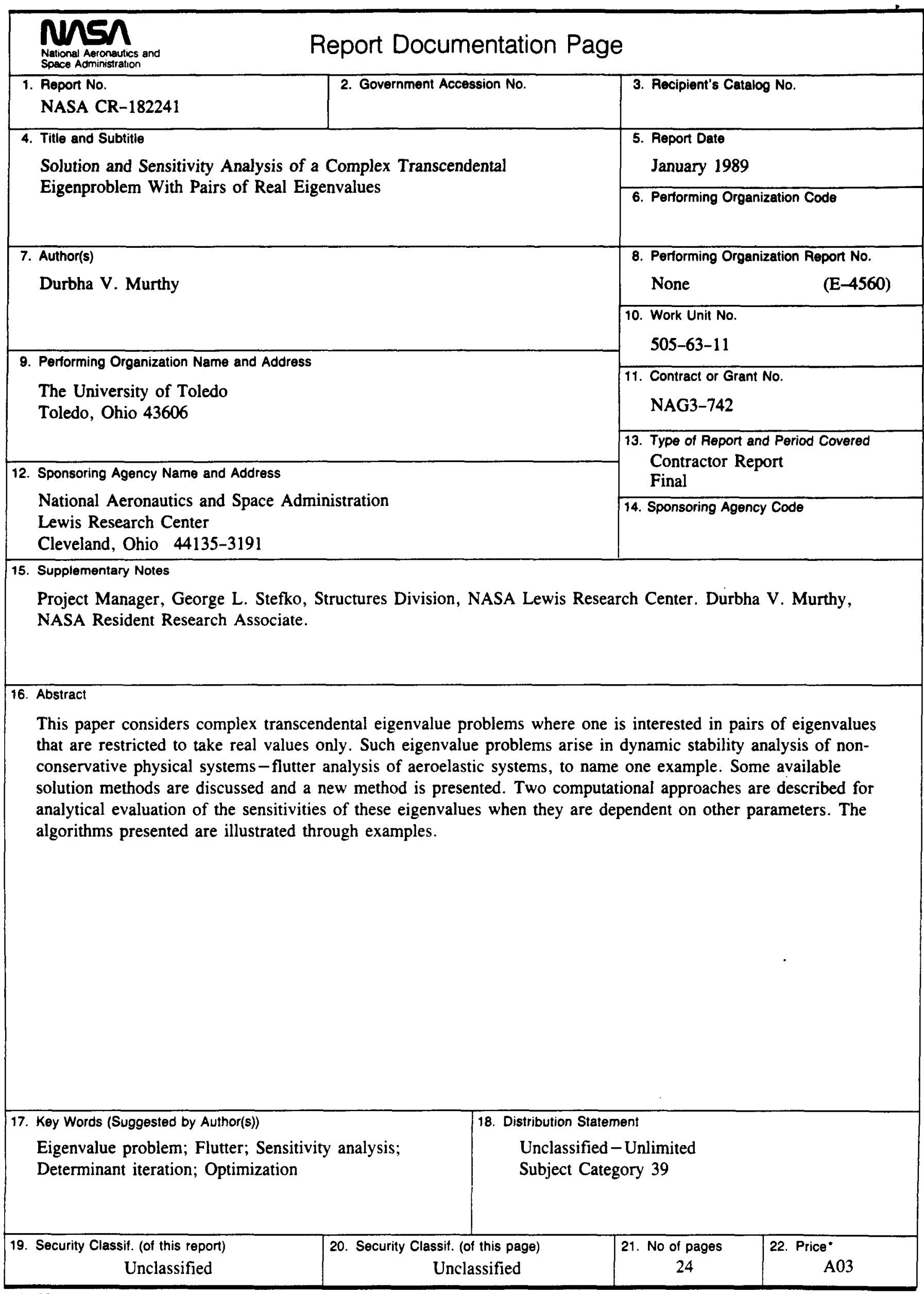

NASA FOAM 1626 OCT $86 \quad$ *For sale by the National Technical Information Service, Springfield, Virginia 22161 